\title{
Defeitos de fechamento do tubo neural e fatores associados em recém-nascidos vivos e natimortos
}

\author{
Neural tube defects and associated factors in liveborn and stillborn infants
}

\author{
Marcos J.B. Aguiar ${ }^{1}$, Ângela S. Campos ${ }^{2}$, Regina A.L.P. Aguiar ${ }^{3}$, \\ Ana Maria A. Lana ${ }^{4}$, Renata L. Magalhães ${ }^{5}$, Luciana T. Babeto ${ }^{5}$
}

\section{Resumo}

Objetivos: avaliar a prevalência e fatores associados aos defeitos de fechamento do tubo neural em recém-nascidos vivos e natimortos, na maternidade do Hospital das Clínicas da UFMG, entre 01/08/1990 e 31/07/2000.

Métodos: trata-se de trabalho descritivo, baseado em banco de dados, seguindo normas do Estudo Colaborativo Latino-Americano de Malformações Congênitas (ECLAMC). São confeccionadas fichas dos recém-nascidos vivos e natimortos com anomalias congênitas, com descrição morfológica, resultados de necropsia, exames complementares, história familiar, social, da gravidez, e outros dados clínicos. Cada recém-nascido vivo malformado dá origem a um controle, do mesmo sexo, sem malformações. Os recém-nascidos vivos e natimortos com defeitos de fechamento do tubo neural, no período, foram classificados de acordo com o defeito apresentado e com a presença ou ausência de outros defeitos associados. Foram comparados sexo, peso, idade e paridade materna com os demais recém-nascidos vivos e natimortos do período. A análise estatística utilizou o programa EpiInfo 6.0 .

Resultados: a prevalência dos defeitos de fechamento do tubo neural foi de 4,73:1.000 partos (89:18.807), tendo sido maior entre os natimortos (23,7:1.000) que entre os recém-nascidos vivos (4,16:1.000), $\mathrm{p}<0,001$. Os defeitos de fechamento do tubo neural foram mais freqüentes entre os recém-nascidos vivos de baixo peso $(\leq 2.500 \mathrm{~g})$, $\mathrm{p}<0,001$, e menos freqüentes entre os filhos de multíparas ( $>3$ gestações), $p=0,007$. Não houve associação com sexo ou idade materna. Entre os natimortos não houve associação com sexo, peso, paridade ou idade materna. Os defeitos de fechamento do tubo neural mais freqüentes foram mielomeningocele $(47,2 \%)$, anencefalia $(26,9 \%)$ e encefalocele $(16,9 \%)$. Os defeitos se encontravam como anomalia isolada em $71,1 \%$ dos recém-nascidos vivos e $38,5 \%$ dos natimortos; faziam parte de uma síndrome em 9,2\% dos recém-nascidos vivos e em 7,7\% dos natimortos.

Conclusão: a prevalência dos defeitos de fechamento do tubo neural foi maior do que a descrita na literatura internacional e na literatura latino-americana.

J Pediatr (Rio J) 2003;79(2):129-34: anormalidades, defeitos do tubo neural, anencefalia, encefalocele, mielomeningocele.

\begin{abstract}
Objectives: to evaluate the prevalence and factors associated to neural tube defects in liveborn and stillborn infants delivered at the Hospital das Clínicas, UFMG, from January 8, 1999 to July 31, 2000.

Methods: this is a descriptive study, based on a database, according to the Latin-American Collaborative Study of Congenital Malformation (ECLAMC) rules. Reports on liveborn and stillborn infants with congenital anomalies were prepared including information about morphological description, necropsy results, complementary exams, family, social and pregnancy histories and other clinical data. Each malformed liveborn infant originated a control of the same sex, without malformations. The liveborn and stillborn infants with neural tube defects delivered during that period were classified according to their defect and the presence or absence of associated defects. The liveborn and stillborn infants with neural tube defects were compared to newborns without neural tube defects according to their weight and sex and their mother's age and parity. Epi-Info 6.0 Program was used for the statistical analysis of the results.

Results: the prevalence of neural tube defects was 4.73 to 1,000 deliveries $(89: 18,807)$; it was significantly higher among stillborn infants $(23.7: 1,000)$ than among liveborn infants $(4.16: 1,000), \mathrm{p}<0.001$. Neural tube defects were more often found among low weight liveborn infants $(\leq 2,500 \mathrm{~g}), \mathrm{p}<0.001$ and less frequently among women who had had more than three gestations, $\mathrm{p}=0.007$. No association was found regarding newborn's sex or maternal age. There was no association with newborn's sex and weight, maternal parity or age among stillborn infants. The most common neural tube defects were myelomeningocele (47.2\%), anencephaly (26.9\%) and encephalocele (16.9\%). The defects were found as isolated anomalies in $71.1 \%$ of the liveborn and $38.5 \%$ of the stillborn infants; they were part of a syndrome in $9.2 \%$ (liveborn) and $7.7 \%$ (stillborn).

Conclusion: the neural tube defect prevalence found in this study was higher than the one described in international and Latin-American
\end{abstract} literature.

J Pediatr (Rio J) 2003;79(2):129-34: abnormalities, neural tube defects, anencephaly, encephalocele, myelomeningocele.

1. Professor Adjunto, Doutor, do Departamento de Pediatria da Faculdade de Medicina da UFMG.

2. Médica do Hospital das Clínicas da UFMG.

3. Professora Adjunta, Doutora, do Departamento de Ginecologia e Obstetrícia da Faculdade de Medicina da UFMG.

4. Professora Adjunta, Doutora, do Departamento de Anatomia Patológica da Faculdade de Medicina da UFMG.

5. Acadêmicas de Medicina da Faculdade de Medicina da UFMG. 


\section{Introdução}

Os defeitos do fechamento do tubo neural (DFTN) são malformações congênitas freqüentes que ocorrem devido a uma falha no fechamento adequado do tubo neural embrionário, durante a quarta semana de embriogênese $\mathrm{e}^{1,2}$. Apresentam um espectro clínico variável, sendo os mais comuns a anencefalia e a espinha bífida ${ }^{3}$.

A anencefalia é a ausência completa ou parcial do cérebro e do crânio. A espinha bífida é um defeito de fechamento ósseo posterior da coluna vertebral. O defeito pode ser recoberto por pele essencialmente normal (espinha bífida oculta), ou associar-se com uma protrusão cística, podendo conter meninges anormais e líquido cefalorraquidiano - meningocele; ou elementos da medula espinhal e/ou nervos - mielomeningocele. Outra forma clínica encontrada é a encefalocele, na qual o cérebro e as meninges herniam-se através de um defeito na calota craniana ${ }^{4}$. Aproximadamente $20 \%$ das crianças afetadas por DFTN apresentam algum outro defeito congênito associado ${ }^{5}$.

Embora varie consideravelmente nas diversas regiões geográficas, a incidência dos DFTN, de uma maneira geral, se situa em torno de 1:1.000 nascimentos vivos ${ }^{5,6}$. O risco de recorrência em futuras gravidezes de um casal que teve um filho com DFTN é cerca de 25 a 50 vezes maior que o risco da população em geral, se situando entre 4 e $5 \%$ 6-8.

Embora os DFTN apresentem etiologia heterogênea e sejam descritos diversos mecanismos em sua gênese, a maioria dos casos é atribuída à interação entre vários genes e fatores ambientais, o que é denominado de herança multifatorial $^{9}$.

A forma como o mecanismo genético atua ainda não está bem esclarecida, mas há fortes evidências do seu envolvimento. Estudos demonstraram que parentes de primeiro grau possuem maior risco de DFTN que os parentes mais distantes. Outra linha de evidência é a presença de DFTN em diversas síndromes genéticas, como na síndrome de Meckel-Gruber, na síndrome de Waardenburg e nas Trissomias dos cromossomos 13 e $18^{6}$.

Propõe-se que vários genes estejam envolvidos no fechamento do tubo neural. Alguns desses genes podem conferir um forte componente genético, enquanto outros podem produzir apenas um pequeno efeito ou interagir com outros genes. Os genes candidatos mais estudados são aqueles associados com o metabolismo do ácido fólico, como o 5,10 metileno-tetra-hidrofolato-redutase. Diversos pesquisadores têm relatado uma freqüência significativamente aumentada de homozigotos da mutação C677T deste gene em afetados, bem como em suas mães ${ }^{6,10}$.

Além de genes, diversos fatores ambientais parecem também envolvidos na etiologia dos DFTN. O ácido fólico é o fator de risco para os DFTN mais importante identificado até hoje. $\mathrm{O}$ exato mecanismo como o ácido fólico está envolvido na embriogênese do tubo neural é ainda desconhecido. Sabe-se que a suplementação periconcepcional e durante o primeiro trimestre de gravidez tem reduzido tanto o risco de ocorrência como risco de recorrência para os DFTN em cerca de 50 a $70 \%$. O CDC recomenda para mulheres que estejam planejando uma gravidez com história familiar negativa de DFTN a dose de $0,4 \mathrm{mg} /$ dia. Para mulheres com alto risco, com história prévia de filhos com DFTN, a recomendação é de $4 \mathrm{mg} / \mathrm{dia}^{5,6,11-14}$.

Outros agentes teratogênicos possivelmente envolvidos como fatores de risco para os DFTN são diabetes mellitus materno, uso de ácido valpróico para tratamento de epilepsia durante a gestação, obesidade materna, deficiência de zinco e hipertermia ${ }^{1,7,15}$.

Os DFTN são determinantes importantes de morbimortalidade perinatal. Todas as crianças anencéfalas são natimortas ou morrem pouco tempo após o nascimento. Crianças com meningocele e mielomeningocele têm uma taxa de sobrevivência maior, geralmente devida a extensivos tratamentos médicos e cuidados cirúrgicos. O seu risco de morte depende da gravidade da lesão e de outros fatores, como disponibilidade de recursos médicos e cirúrgicos ${ }^{5}$. Já a espinha bífida oculta pode evoluir de forma assintomática, durante toda a vida.

A criança com menigocele ou mielomeningocele pode apresentar incapacidades crônicas graves, como paralisia de membros, hidrocefalia, deformação de membros e da coluna vertebral, disfunção vesical, intestinal, sexual e dificuldade de aprendizagem, com risco de desajuste psicossocial. Nos Estados Unidos, os gastos, por toda a vida, com cada criança nascida com espinha bífida, é estimado em aproximadamente US\$294.000,005 .

Devido à gravidade dos DFTN e sua alta morbimortalidade, tornam-se muito importantes o aconselhamento genético, a suplementação dietética com ácido fólico e o diagnóstico pré-natal das malformações do tubo neural. Este pode ser feito através do ultra-som durante a gestação e dosagem de alfa-feto proteína, no líquido amniótico, cujos valores estarão aumentados, através da amniocentese, entre a $14^{\mathrm{a}}$ e $16^{\mathrm{a}}$ semanas de gestação.

Dispomos de poucos dados sobre o impacto dos DFTN entre nós, o que tem grande importância na definição da estratégia mais adequada para a redução da sua incidência.

Desde 1967, o Estudo Colaborativo Latino-Americano de Malformações Congênitas (ECLAMC) realiza pesquisas clínicas e epidemiológicas das anomalias congênitas e suas causas, em nascimentos hospitalares, cobrindo mais de 100 hospitais, de nove países da América do Sul, diversos deles localizados no Brasil. Em 1994, foi reconhecido pela Organização Mundial de Saúde como Centro Colaborador para a Prevenção das Malformações Congênitas. Já foram examinados mais de quatro milhões de nascimentos nos seus 35 anos de existência. A prevalência de defeitos de fechamento do tubo neural entre esses nascimentos é de cerca de 1,5:1.000 nascimentos ${ }^{7}$. A Maternidade do Hospital das Clínicas da UFMG está vinculada ao ECLAMC desde $1^{\circ}$ de agosto de 1990. 


\section{Objetivos}

Avaliar a prevalência e fatores associados aos diversos tipos de defeitos de fechamento do tubo neural em 18.807 partos consecutivos, em dez anos (agosto de 1990 a julho de 2000) de monitoração de defeitos congênitos, na Maternidade do Hospital das Clínicas da UFMG (HC-UFMG).

\section{Material e métodos}

Desde a vinculação da Maternidade do Hospital das Clínicas da UFMG ao Estudo Colaborativo Latino-Americano de Malformações Congênitas (ECLAMC), foi criado um banco de dados com os recém-nascidos vivos (RNV) e mortos (RNM) com malformações congênitas e seus controles. É preenchida uma ficha, padronizada pelo ECLAMC, de todo RNV ou RNM com malformações, na qual são anotados a descrição morfológica, dados de necropsia, resultados de exames complementares, intercorrências durante a gravidez, história familiar de malformações congênitas, consangüinidade, sexo e peso do RN, gemelaridade, paridade e idade maternas, tipo de parto, apresentação e aspectos socioeconômicos. Cada RNV malformado dá origem a um RNV controle, definido como sendo o primeiro nascido vivo após o caso, com o mesmo sexo, sem malformações. De cada RNV controle são colhidos os mesmos dados. Para os RNM não são feitos controles. Mensalmente são confeccionados informes de nascimentos com o número de RNV e RNM classificados por sexo, peso, agrupado em intervalos de 500g, idade materna, agrupada em intervalos de 5 anos, paridade materna e gemelaridade.

Foi realizado um estudo descritivo, através da análise deste banco de dados. Foram revisados os registros de anomalias e os informes mensais de nascimento, sendo identificados todos os RNV e RNM com DFTN. Comparamos sexo, peso, idade e paridade maternas desses pacientes com os demais RNV e RNM nascidos no hospital no mesmo período estudado. Os casos de DFTN encontrados foram identificados conforme sua apresentação clínica (anencefalia, encefalocele, meningocele, mielomeningocele) sendo classificados como malformações isoladas, como componente de síndromes e/ou sequiências, associados com outras anomalias no sistema nervoso central ou em outros órgãos e sistemas, e como polimalformados (associados com malformações múltiplas sem diagnóstico sindrômico). A análise estatística dos dados foi realizada no programa Epi-Info $6.0^{17}$, com utilização dos testes do qui-quadrado e exato de Fisher, de acordo com a indicação.

\section{Resultados}

No período estudado, ocorreram 18.807 partos, sendo 18.258 de RNV e 549 de RNM. Foram diagnosticados 89 casos de DFTN (prevalência de 4,73:1.000). Setenta e seis casos ocorreram entre RNV (prevalência de 4,16:1.000), e 13 casos entre RNM (prevalência de 23,7:1.000). A prevalência dos DFTN foi significativamente maior entre os RNM ( $\mathrm{p}<0,001)$.

Entre os RNV, os DFTN foram mais freqüentes naqueles de baixo peso $(\leq 2.500 \mathrm{~g}), \mathrm{p}<0,001$, e menos freqüente entre os filhos de multíparas (mais de 3 gestações), $\mathrm{p}=0,007$ (Tabela 1). Não houve associação com sexo ou idade materna.

Entre os RNM, não houve associação com nenhum dos fatores investigados: sexo, peso, paridade e idade materna.

Dos 89 casos de DFTN, 42 (47,2\%) foram de mielomeningocele, $24(26,9 \%)$ de anencefalia, $15(16,9 \%)$ de encefalocele, cinco $(5,6 \%)$ de meningocele e três $(3,4 \%)$ de associação entre dois tipos de DFTN (dois casos de anencefalia + mielomeningocele, e um caso de encefalocele + meningocele) (Tabela 2).

Tabela 1 - Fatores associados aos defeitos de fechamento do tubo neural

\begin{tabular}{lccrc}
\hline Característica & DFTN (+) & DFTN (-) & Totais & Valor de p \\
\hline Peso ao nascer & & & & \\
$\leq 2.500 \mathrm{~g}$ & 35 & 3.564 & 3.599 & \\
$>2.500 \mathrm{~g}$ & 41 & 14.528 & 14.569 & $<0,001$ \\
Não especificados: $90 \mathrm{RNV}$ & & & & \\
Número de gravidezes maternas & & & & \\
$\leq 3$ & 69 & 13.986 & 14.055 & \\
$>3$ & 7 & 4.173 & 4.180 & 0,007 \\
Não especificados: 23 RNV & & & & \\
\hline
\end{tabular}

Não foram significativos: sexo e idade materna.

DFTN: defeitos de fechamento do tubo neural, RNV: recém-nascidos vivos. 
Tabela 2 - Tipos de defeitos de fechamento do tubo neural em recém-nascidos vivos

\begin{tabular}{lcc}
\hline Tipo de DFTN & Número & Percentagem \\
\hline Meningomielocele & 42 & $47,2 \%$ \\
Anencefalia & 24 & $26,9 \%$ \\
Encefalocele & 15 & $16,9 \%$ \\
Meningocele & 5 & $5,6 \%$ \\
Dois defeitos & 3 & $3,4 \%$ \\
Total & 89 & $100 \%$ \\
\hline
\end{tabular}

DFTN: defeitos de fechamento do tubo neural.

Entre os 76 RNV com DFTN, 54 (71,1\%) apresentavam o defeito como anomalia única (30 casos com mielomeningocele, 11 casos com anencefalia, nove casos com encefalocele e quatro casos com meningocele). Sete RNV $(9,2 \%)$ apresentavam o defeito como parte de uma síndrome ou associação (três casos de síndrome de Meckel-Gruber, dois de síndrome do cordão curto, um de trissomia do cromossomo 18 e um de associação VATER). Sete $(9,2 \%)$ apresentavam associadas aos DFTN anomalias em outros órgãos e sistemas, com acometimento principalmente dos sistemas geniturinário, endócrino e locomotor. Em cinco casos $(6,6 \%)$, os DFTNs faziam parte de um padrão de anomalias múltiplas, sem diagnóstico sindrômico definido. Em três casos $(3,9 \%)$, os DFTN estavam associados com outras anomalias do sistema nervoso central (Tabela 3).

Entre os 13 RNM, cinco (38,5\%) apresentavam o DFTN como malformação isolada, todos com anencefalia. Em outros cinco casos $(38,5 \%)$, houve associação de anencefalia com malformações em outros sistemas, principalmente cardiovascular e digestivo. Em dois casos $(15,4 \%)$ foram encontradas anencefalia e mielomeningocele, sem outras malformações. Em um caso (7,7\%), o DFTN encontrado fazia parte da síndrome de Meckel-Gruber (Tabela 3).

\section{Discussão}

A prevalência dos DFTN encontrada em nosso hospital foi alta, comparada com a descrita na literatura ${ }^{5,6}$ e com aquela registrada pelo ECLAMC para a América do Sul ${ }^{7}$. Dois fatores podem ter contribuído para esta alta prevalência: a maternidade do Hospital das Clínicas da UFMG é um centro de referência local e regional em medicina fetal, para onde são encaminhados os casos de gravidez de alto risco e aqueles em que se suspeita, ou mesmo se diagnosticou, uma anomalia fetal, e o fato de o Hospital das Clínicas contar com um serviço de correção cirúrgica das meningoceles, mielomeningoceles e encefaloceles.

Com o objetivo de reduzir a incidência de DFTN, diversos países vêm preconizando a adição de ácido fólico em alimentos consumidos em grande escala pela população. Nos Estados Unidos, isto levou a um declínio de aproximadamente $19 \%$ na incidência de DFTN ${ }^{16}$. O Chile foi o primeiro país da América do Sul a adicionar ácido fólico a alimentos ${ }^{7}$. Considerando os custos do tratamento das meningoceles, mielomeningoceles e encefaloceles, acreditamos que é tempo de se avaliar a relação custos/benefícios da introdução de ácido fólico em alimentos de alto consumo em nosso país. Os resultados que venham a ser observados no $\mathrm{Chile}^{7}$ serão de especial valia para este objetivo.

Os achados deste trabalham também validam a utilização de ultra-sonografia e de testes bioquímicos para a triagem e diagnóstico pré-natal dos DFTN.

Encontramos uma maior prevalência dos DFTNs em RNV com baixo peso, o que é descrito na literatura ${ }^{4}$. Esta prevalência aumentada pode ser resultado do efeito das próprias anomalias no crescimento fetal, ou se dever ao maior risco de malformações nesse grupo de recém-nascidos.

A menor prevalência dos DFTN entre os RNV filhos de multíparas verificada em nossa amostra é concordante com o descrito em alguns trabalhos ${ }^{4,18}$.

Tabela 3 - Apresentação dos defeitos de fechamento do tubo neural em recém-nascidos vivos

\begin{tabular}{lcc}
\hline Forma de apresentação & RNV & RNM \\
\hline $\begin{array}{l}\text { DFTN como defeito único } \\
\text { DFTN como sinal (em uma síndrome) }\end{array}$ & $54(71,1 \%)$ & $5(38,5 \%)$ \\
$\begin{array}{l}\text { DFTN associado a malformações } \\
\text { de outros órgãos e sistemas }\end{array}$ & $7(9,2 \%)$ & $1(7,7 \%)$ \\
$\begin{array}{l}\text { DFTN em um padrão de anomalias múltiplas } \\
\text { sem diagnóstico sindrômico definido }\end{array}$ & $7(9,2 \%)$ & $5(38,5 \%)$ \\
$\begin{array}{l}\text { DFTN associado a outras malformações } \\
\text { do sistema nervoso central }\end{array}$ & $5(6,6 \%)$ & 0 \\
\end{tabular}

DFTN: defeitos de fechamento do tubo neural, RNV: recém-nascidos vivos, RNM: natimortos. 
Há relatos na literatura de que os DFTNs sejam mais comuns no sexo feminino e de que haja uma maior suscetibilidade para essas malformações em mães com idade entre 20 e 24 anos $^{4}$; porém, em nossa amostra, não foram encontradas tais associações.

A maior prevalência de DFTN observada entre os RNM reflete a prevalência aumentada de malformações congênitas entre natimortos, quando comparados com recém-nascidos vivos ${ }^{18}$.

\section{Referências bibliográficas}

1. Northrup H, Volcik KA. Spina bifida and other neural tube defects. Curr Probl Pediatr 2000;30(10):313-32.

2. Gelineau-van Waes J, Finnell RH. Genetics of neural tube defects. Semin Pediatr Neurol 2001;8(3):160-4.

3. Drugan A, Weissman A, Evans MI. Screening for neural tube defects. Clin Perinatol 2001;28(2):279-87.

4. Hunter GWA. Brain and Spinal Cord. In: Stevenson ER, Hall JG, Goodman RM, editores Human malformations and related anomalies. Oxford: Oxford University Press; 1993.p.109-31.

5. Botto LD, Moore CA, Khoury JM, Erickson JD. Neural tube defects - Review articles. Medical Progress 1999;341(20): 1509-17.

6. Melvin CE, George TM, Worley G, Franklim A, Mackey J, Viles $\mathrm{K}$, et al. Genetic studies in neural tube defects. Pediatr Neurosurg 2000;32:1-9.

7. Nazer-HJ, López Camelo JS, Castilla EE. ECLAMC: Estudio de 30 años de vigilancia epidemiológica de defectos de tubo neural en Chile y en Latino América. Rev Med Chil 2001;129:531-9.

8. Speer MC, McLone D, Nye J, Worley G, Melvin EC, Viles KD, et al. NTD Collaborative Group. Possible interaction of genotypes at cystathionine $B$-synthase and Methylenetetrahydrofolate reductase (MTHFR) in neural tube defects. Clin Genet 1999;56:142-4.
Como descrito na literatura, a anencefalia foi o DFTN predominante entre os natimortos ${ }^{5}$. Apenas um natimorto, com síndrome de Meckel, apresentava um DFTN diferente da anencefalia.

Os DFTNs apresentaram-se principalmente como malformações isoladas, sendo a mielomeningocele e a anencefalia os mais comuns entre os RNV. Entre os RNM, a anencefalia foi o defeito mais freqüente.
9. On Line Mendelian Inheritance in Man, OMIM ${ }^{\mathrm{TM}}$. Johns Hopkins University, Baltimore, MD. MIM NUMBERS: \# 601634 e \# 601635 [site na Internet]. Disponível em: URL: http:// www.ncbi.nlm.nih.gov/omim/. Acessado 20 de março de 2003.

10. Richter B, Stegmann K, Roper B, Boddeker I, Ngo ET, Koch MC. Interaction of folate and homocysteine pathway genotypes evaluated in susceptibility to neural tube defects (NTD) in a German population. J Hum Genet 2001;46(3):105-9.

11. Harris MJ, Juriloff DM. Mini-review: toward understanding mechanisms of genetic neural tube defects in mice. Teratology 1999;60:292-305.

12. MRC Vitamin Study Research Group. Prevention of neural tube defects: Results of the Medical Research Council Vitamin Study. Lancet 1991;338:131-7.

13. Abramsky L, Botting B, Chapple J, Stone D. Has advice on periconceptional folate supplementation reduced neural tube defects? Lancet 1999;354:998-9.

14. Krishnaswamy K, Madhavan Nair K. Importance of folate in human nutrition. Br J Nutr 2001;85 (Suppl 2):115-24.

15. Kozma C. Valproic acid embryopathy: report of two siblings with further expansion of the phenotypic abnormalities and a review of the literature. Am J Med Genet 2001;98(2):168-75.

16. Honein MA, Paulozzi LJ, Mathews TJ, Erickson JD, Wong LY. Impact of folic acid fortification of the US food supply on the occurrence of neural tube defects. JAMA 2001;285(23):3022-3. 
17. Dean AG, Dean JA, Coulombier D, Brendel KA, Smith DC, Burton AH, et al. Epi-Info version 6: a Word processing database and statistics program for epidemiology on microcomputers, 1994, Centers for Disease Control and Prevention, Atlanta, Georgia, USA.

18. Winter RM, Knowles SAS, Bieber FR, Baraitser M. The malformed fetus and stillbirth. A diagnostic approach. Chichester: John Willey \& Sons; 1989.
Endereço para correspondência:

Dr. Marcos José Burle de Aguiar

Rua Timbiras, 659 - ap. 1001 - Funcionários

CEP 30140-060 - Belo Horizonte, MG

Fone: (31) 3274.3453

E-mail: aguiarmr@terra.com.br 